\title{
Schema Induction and Analogical Transfer
}

\author{
Mary L. Gick and Keith J. Holyoak \\ University of Michigan
}

\begin{abstract}
An analysis of the process of analogical thinking predicts that analogies will be noticed on the basis of semantic retrieval cues and that the induction of a gencral schema from concrete analogs will facilitate analogical transfer. These predictions were tested in experiments in which subjects first read one or more stories illustrating problems and their solutions and then attempted to solve a disparate but analogous transfer problem. The studies in Part I attempted to foster the abstraction of a problem schema from a single story analog by means of summarization instructions, a verbal statement of the underlying principle, or a diagrammatic representation of it. None of these devices achieved a notable degree of sucess. In contrast, the experiments in Part II demonstrated that if two prior analogs were given, subjects often derived a problem schema as an incidental product of describing the similarities of the analogs. The quality of the induced schema was highly predictive of subsequent transfer performance. Furthermore, the verbal statements and diagrams that had failed to facilitate transfer from one analog proved highly beneficial when paired with two. The function of examples in learning was discussed in light of the present study.
\end{abstract}

Analogy pervades thought. When a John Donne proposes that "no man is an island," we feel an intuitive grasp of the interconnectedness of human relations. When a William Harvey compares a biological organ to a water pump, a productive scientific model of blood circulation is created; in addition, the meaning of "pump" may take on a new, more abstract form. When a student is told that the atom resembles a miniature solar system, a complex new concept may take root in the learner's mind. To make the novel seem familiar by relating it to prior knowledge, to make

This paper is largely based on a PhD dissertation completed by Gick (Note 1) under the direction of Holyoak, together with additional collaborative experiments and analyses. The work benefitted from the guidance of the members of the dissertation committee: John Jonides, Manfred Kochen, David Krantz, and substitute member John Holland. Patricia Cheng provided incisive criticisms of an early draft; a subsequent draft benefitted from the reviews of Dedre Gentner, Earl Hunt, and an anonymous referee. Terra Albert, Holly Brewer, Tim Carroll, Teresa Frankovich, and Michael Smith ably assisted in testing subjects. Susan Petersen and Jean Schtokal assisted with both subject testing and scoring of data. Experiment 4 was reported at the 20th meeting of the Psychonomic Society, Phoenix, November 1979. This research was supported by a University of Michigan Rackham Dissertation Grant to Gick, and by National Institute for Mental Health Grant 5-R01MH332878-03 and National Institute for Mental Health Research Scientist Development Award 1-K02-MH00342-02, both to Holyoak. Reprint requests may be sent to K. Holyoak, University of Michigan, Human Performance Center, 330 Packard Road, Ann Arbor, MI 48104 . 
the familiar seem strange by viewing it from a new perspective-these are fundamental aspects of human intelligence that depend on the ability to reason by analogy. This ability is used to construct new scientific models, to design experiments, to solve new problems in terms of old ones, to make predictions, to construct arguments, and to interpret literary metaphors. (See Miller, 1979, for a recent defense of the classical view that metaphor should be analysed in terms of analogy.)

The essence of analogical thinking is the transfer of knowledge from one situation to another by a process of mapping - finding a set of one-toone correspondences (often incomplete) between aspects of one body of information and aspects of another. A central psychological issue concerns the mechanisms underlying analogical transfer. Reasoning by analogy typically implies a comparison of two concepts ("analogs") at the same (usually quite concrete) level of abstraction (e.g., the heart and a water pump). However, a similar mapping process may be required to compare a specific concept to a more general schema (e.g., the heart and the abstract concept of "pump"). Furthermore, mapping may also be involved in the induction of schemas from examples (e.g., learning the abstract sense of "pump" by comparing hearts and water pumps). Such a close relationship between the processing of concrete analogs and general schemas is supported both by experimental evidence (Schustack \& Anderson, 1979) and computational analysis (Winston, 1980). ${ }^{1}$

The present study was designed to investigate the mechanisms that govern analogical transfer between semantically disparate problems. The central empirical questions concern how analogies are noticed and then applied to generate solutions to novel problems. In addressing these questions the experiments explore the induction of a problem schema from concrete analogs and the role of the schema in fostering subsequent transfer. To provide a context for the empirical work, we will first briefly review our previous research on analogical problem solving and then provide a theoretical analysis of the nature of analogy and its relationship to schema induction. In addition to generating predictions regarding the determinants of analogical transfer, this analysis may serve to clarify the concept of "schema," which has been widely applied in cognitive models, but also widely criticized for its vagueness.

\section{BACKGROUND RESEARCH}

Most psychological research on analogical reasoning has used fourterm “proportions" as stimuli (i.e., A:B::C:?; for a review see Sternberg, 1977). The adequacy of the proportion framework as a model of analogical thinking has been questioned by Hesse (1966) and Weitzenfeld and Klein

\footnotetext{
${ }^{1}$ The present distinction between a general schema and a specific analog is close to that between the first and second senses of the term "model" as it is used by Hesse (1966).
} 
(Note 2). A major limitation is that such stimuli obviate any need for the subject to spontaneously notice the analogy, which is often a prerequisite for successful transfer in realistic problem situations. A few studies have investigated the use of analogies to solve problems. For example, Gentner and Gentner (in press) have demonstrated that alternative analogies, known by subjects prior to the experiment, produce systematically varying patterns of difficulty among types of electricity problems. Other studies have indicated that potential analogies presented in the experimental context are often not used (Reed, Ernst, \& Banerji, 1974) or even noticed, especially when the problem analogs are from different semantic domains (Duncker, 1926; Gick \& Holyoak, 1980). We will discuss the Gick and Holyoak study in more detail, since it led directly to the present investigation. We had subjects attempt to solve Duncker's (1945) "radiation problem," which was the primary problem used in the present study:

Suppose you are a doctor faced with a patient who has a malignant tumor in his stomach. It is impossible to operate on the patient, but unless the tumor is destroyed the patient will die. There is a kind of ray that can be used to destroy the tumor. If the rays reach the tumor all at once at a sufficiently high intensity, the tumor will be destroyed. Unfortunately, at this intensity the healthy tissue that the rays pass through on the way to the tumor will also be destroyed. At lower intensities the rays are harmless to healthy tissue, but they will not affect the tumor either. What type of procedure might be used to destroy the tumor with the rays, and at the same time avoid destroying the healthy tissue?

Prior to their attempt to solve the radiation problem, our subjects often read a story about an analogous military problem and its solution (see "The General" in Appendix II). In this story a general wishes to capture a fortress located in the center of a country. There are many roads radiating outward from the fortress. All have been mined so that while small groups of men can pass over the roads safely, any large force will detonate the mines. A full-scale direct attack is therefore impossible. The general's solution is to divide his army into small groups, send each group to the head of a different road, and have the groups converge simultaneously on the fortress. As you may have already noticed, there is an analogous "convergence" solution to the radiation problem. The doctor could direct multiple low-intensity rays toward the tumor simultaneously from different directions, so that the healthy tissue will be left unharmed, but the effects of the low-intensity rays will summate and destroy the tumor. ${ }^{2}$

${ }^{2}$ In our earlier paper (Gick and Holyoak, 1980) we referred to this as the "dispersion" solution, emphasizing the initial division of the single large force into several small ones and their dispersal to multiple locations. However, in some forms of the problem a single large force may not initially exist (see "Red Adair" in Appendix II), making our original name for the solution quite misleading. Calling it the convergence solution emphasizes its central property, the convergence of multiple forces on the target. 
A paradigm for investigating analogical problem solving must satisfy two basic requirements. First, a relevant known analog must be available to the subject. Provision of a story analog prior to the radiation problem fulfilled this prerequisite. Second, the target problem must be sufficiently novel and challenging that an analogy could potentially be useful. Subjects are unlikely to bother to apply an analogy if a solution to the target problem is already known or can be easily achieved by means-ends analysis. The radiation problem, due to its ill-defined nature, meets the second requirement. The problem admits of a variety of potential solution plans, and we were able to exploit this feature to demonstrate the influence of an analogy. By varying the solution provided in the military story, we were able to selectively facilitate the discovery of various alternative potential solutions to the radiation problem. The influence of the analogy was very pronounced. For example, about $75 \%$ of those subjects who received the appropriate military analog produced the convergence solution to the radiation problem, as compared to less than $10 \%$ of control subjects who did not receive the analogy.

The above results were obtained when subjects were given an explicit hint to use the prior story to help solve the radiation problem. The hint was quite nonspecific, as subjects were told nothing about how the story might help; however, it obviated any need for subjects to spontaneously notice the potential analogy. In further experiments we attempted to assess subjects' ability to notice the analogy separately from their ability to subsequently apply it to generate an analogous solution to the target problem. The study of noticing raises some tricky methodological issues. Ideally, one would like to provide an ecologically natural context for the prior analog; however, the range of such contexts is not readily delimited. Subjects must be led to process the analog in such a way that the information will be potentially retrievable when they later encounter the target problem. Task demands should neither make it obvious to subjects that they should use the analogy, nor preclude the possibility of their noticing its relevance.

To approximate the above requirements we had subjects first memorize the military story analog in the guise of a study of story recall and then immediately go on to work on the radiation problem, without any hint to use the prior story. Under these conditions only about $30 \%$ of our subjects produced the convergence solution, as opposed to about $75 \%$ who produced it when a hint was given. Assuming that about $10 \%$ of subjects would produce the solution in the absence of any analogy (a figure based on several replications), these results indicate that only a third or less of the subjects who could potentially apply the analogy spontaneously noticed it. We found this striking gap between noticing and application quite surprising, although, as noted above, comparable outcomes have 
been obtained in other transfer paradigms (Kohler, 1940; Reed et al., 1974). Our procedure did not involve any deception; subjects were told at the outset that the experiment would have two parts, story recall and then problem solving. The delay between the two tasks was minimal. One might well have supposed that the demand characteristics of being in a psychology experiment would have led virtually all subjects to consider how the first part might be related to the second. Indeed, a participant in one of our earlier experiments had complained to us that giving a hint to use the story was an insult to our subjects' intelligence!

It is thus no easy matter to spontaneously notice an analogy between two superficially dissimilar situations, even in our highly simplified experimental paradigm. The present study investigates factors that influence the likelihood that subjects will notice and apply analogies. To derive specific predictions we first need to analyze the structure of analogy and the relationship between specific analogs and more abstract schemas.

\section{ANALOGICAL THINKING AND SCHEMAS}

\section{Structure of Analogy}

It is important to recognize that the structure of analogy is dictated by its function. In analogical problem solving, one problem and its solution are already known. The analogist notes correspondences between the known problem and a new unsolved one, and on that basis derives an analogous potential solution. More generally, the function of an analogy is to derive a new solution, hypothesis, or prediction; this is done by finding an initial partial mapping between the two analogs and then extending the mapping by retrieving or creating additional knowledge about the analog that was initially less well understood.

Each analog is thus conceptually divided into two parts: that which provides the initial basis for mapping, and that which constitutes the "conclusion." As Hesse (1966) has argued, analogy involves two distinct types of relationships: the "horizontal" mapping relationship between aspects of the two analogs, and the "vertical" relationship between the two parts of a single analog. At the most general level, the latter relationship is between relevant antecedent conditions and their correlated consequences. ${ }^{3}$ In many cases, including analogies between problems, the

\footnotetext{
${ }^{3}$ Metaphors typically produce an initial partial mapping that creates an expectation that additional correspondences can be found, involving correlated aspects of the referent. For example, suppose we assert that "Analogy is a window on the mind." The most salient attribute of a window is probably that it is something one sees through; accordingly, in an appropriate context (such as a psychological report) we might interpret the remark to mean that studying analogy is a way to gain understanding of ("see") the mind. The initial mapping then constitutes an "invitation" to pursue the metaphor further. Thus, one might note that a window is typically a relatively small opening in an opaque wall, suggesting that the
} 
vertical relationships will correspond to causal relations within the person's mental model of each situation (Winston, 1980). For example, certain aspects of an initial problem situation will be viewed as sufficient conditions for the attainment of a particular solution. The vertical and horizontal relationships in an analogy are inextricably linked. For while it is not essential to map all aspects of the two analogs (indeed, this will seldom be possible), those aspects which constitute the causal antecedents of the known outcome obtained in the referent analog must be mappable. If these causal elements cannot be mapped, the putative analogy can be rejected as misleading.

\section{Analogical Mapping and Schema Induction}

To understand the process of reasoning by analogy and the relationship between an analog and a schema, it is necessary to examine the concept of "mapping" in some detail. While many important questions about the mapping process must remain unanswered, several general points deserve emphasis. First, mappings may involve aspects of the analogs that have never been explicitly presented to the analogist. Consequently, the various inference processes required for everyday understanding will often play important roles in analogical thinking. Second, the mapping process will often involve a search for "alternative views" of one of the analogs (Moore \& Newell, 1973; Schon, 1979). For example, in order to use the military story to help solve the radiation problem, subjects must presumably view the story as a problem and its solution, rather than as, say, an anecdote about a populist hero. In addition, mappings can be defined at multiple levels of abstraction, which may correspond to "macrostructures" in the sense of Kintsch and van Dijk (1978) and van Dijk (1980). The optimal level of representation will be that which maximizes the degree of correspondence between causally relevant features of the analogs. Finally, since mapped elements (relations and properties and their arguments) are typically similar but not identical, they must be decomposable into identities and differences (Hesse, 1966; Tversky, 1977).

To make our discussion more concrete, we present in Table 1 an informal description of the mapping relations between the military story and the radiation problem. The correspondences are stated at a fairly abstract level of macrostructure (for a description at a more concrete level, see Gick \& Holyoak, 1980). We assume that each of the two analogs is an instance of a very general "problem schema," which is organized hierar-

mind is generally difficult to study. Furthermore, a small window often allows only partial view of what lies beyond, intimating that analogy may provide limited and selective insights, and so on. The interpretation of metaphor thus seems to begin with detection of salient initial correspondences, followed by the construction of others (Jaynes, 1976, Chap. 2). 
TABLE 1

Correspondences among Two Convergence Problems and Their Schema

Military problem

Initial state

Goal: Use army to capture forces.

Resources: Sufficiently large army.

Constraint: Unable to send entire army along one road.

Solution plan: Send small groups along multiple roads simultaneously.

Outcome: Fortress captured by army.

Radiation problem

Initial state

Goal: Use rays to destroy tumor.

Resources: Sufficiently powerful rays.

Constraint: Unable to administer high-intensity rays from one direction.

Solution plan: Administer low-intensity rays from multiple directions simultaneously.

Outcome: Tumor destroyed by rays.

Convergence schema

Initial state

Goal: Use force to overcome a central target.

Resources: Sufficiently great force.

Constraint: Unable to apply full force along one path.

Solution plan: Apply weak forces along multiple paths simultaneously.

Outcome: Central target overcome by force.

chically into an initial state (goals, available resources, and constraints), a solution plan, and an actual or anticipated outcome of realizing the plan. The problem schema reflects the vertical organization of the analogs, as we discussed earlier; the initial state includes relevant antecedent conditions, of which the solution plan and outcome are consequences. These problem components thus have a natural procedural interpretation as "situation-action" rules (Winston, 1980). The task of the analogist is to construct a partial mapping between the two initial states, which can be used to construct the analogous solution plan and expected outcome for the radiation problem.

At the level of macrostructure assumed in Table 1 the two problems can be completely mapped, i.e., a one-to-one correspondence can be found between their components. For example, the goal of using the army to capture the fortress maps onto the goal of using the rays to destroy the tumor. The basis of such a mapping relation is an abstract "core idea" that both mapped propositions instantiate, which we will term a "mapped identity." (See Holyoak, in press, for a more detailed taxonomy of mapping relations.) In each problem the goal, for example, is to use a force to overcome a central target. At the bottom of Table 1 these mapped iden- 
tities are abstracted and stated as a "convergence schema"; i.e., a representation of the type of problem for which convergence solutions are feasible. Such a schema can be abstracted from the two analogs by "eliminative induction" (Mackie, 1974; see Winston, 1980, for a computational implementation). In essence, the process of schema induction involves deleting the differences between the analogs while preserving their commonalities. ${ }^{4}$

The schema can be viewed as an abstract category that the individual analogs instantiate in different ways. For example, the goal of "overcoming a target" is instantiated as "capturing a fortress" in the military story, and as "destroying a tumor" in the radiation problem. Such domain-specific instantiations of the schema can be termed "structurepreserving differences" between the two analogs (Holyoak, in press). In our example, the military analog can be viewed as a transformation of the convergence schema into concepts appropriate to a military domain, and the medical analog can be viewed as a transformation of the same schema into concepts appropriate to a medical domain. Since the schema is implicitly embedded in each analog, and assuming the mapped identities are causally sufficient to yield the outcome associated with the schema, it follows that structure-preserving differences do not alter the causal relations in the schema. Such differences make the problems analogous rather than identical.

The distinction between mapped identities and structure-preserving differences helps to elucidate the intuitive notion that analogs differ in their similarity to one another. The similarity of any pair of mapped concepts will increase with the extent of the meaning captured by a mapped identity (Tversky, 1977). In general, if the mapped concepts are either identical or instances of a close superordinate concept, the analogs will be very similar (yielding, one might say, a "literal" rather than a "metaphorical" analogy). Thus, a story about a doctor treating a brain tumor with multiple lasers would obviously be more similar to the radiation problem than is our military problem. A "deep" analogy, the sort that captures our admiration, is an analogy between disparate situations in which the essential causal relations are maintained.

Differences between analogs are not always structure preserving. A "structure-violating" difference is one that does not conform to the general transformation relating the schema to its analog (Holyoak, in press). Such differences make the analogy incomplete. While our convergence

\footnotetext{
${ }^{4}$ In the case of metaphor, the formation of a schema from mapped identities, so that the subject and referent can be viewed as instances of a more general concept, may provide a basis for the "interaction view" of metaphorical interpretation (Black, 1962).
} 
analogy is complete at the level of macrostructure represented in Table 1, it is incomplete at more specific levels (see Gick \& Holyoak, 1980). For example, the role of the army in the military story usually corresponds to the role of the rays in the medical problem. But whereas a direct attack on the fortress would endanger the army, direct treatment of the tumor would not endanger the rays. (Rather, it would harm healthy tissue). This violation of role parallelism constitutes a structure-violating difference between the two analogs. Normatively, a structure-violating difference should lead to rejection of the analogy as inappropriate if such a difference alters an element causally necessary for the solution to the known problem (which is not the case for the above example).

In general, increasing the level of representational abstraction will increase the completeness of an analogy, by deleting mismatching details (both structure-preserving and structure-violating differences). Note, however, that greater completeness need not be entirely a virtue. For example, one might claim that any two problems are completely analogous at the level of the abstract problem schema corresponding to the headings used in Table 1. But such abstract analogies will seldom trigger development of a realizable solution procedure. In general, increasing the level of abstraction will at some point delete corresponding identities as well as mismatches, and consequently diminish the similarity of the analogy. A tendency to maximize the completeness of an analogy by moving to a more abstract level of macrostructure may therefore often compete with a tendency to maximize the extensiveness of the mapping between causal relations by moving to a more detailed representational level. As a result, the "optimal" level of representation for successful analogical thinking may typically lie at an intermediate level of abstraction, and it may yield an analogy that is less than complete.

\section{Schemas as Mediators of Analogical Transfer}

The above analysis suggests how analogical transfer may take place. The schema (mapped identities) affords the basis for analogical transfer. However, we can distinguish two conceptually distinct ways in which the schema could be used in solving a problem with reference to information obtained from prior analogs. First, the new problem may be mapped directly with a prior analog to generate the analogous solution. While the mapped identities will mediate the transfer process, the schema need not exist as a separate concept independent of the two analogs (although an independent schema may bc incidentally induced in the course of the mapping process). We will refer to this case as "reasoning from an analog." Second, an independent schema may already have been induced from one or more prior analogs and stored in memory. The person can 
therefore map the new analog directly with the schema in order to construct a solution. This case will be termed "reasoning from a schema."

There are several reasons why our analysis predicts a processing advantage for reasoning for a schema rather than an analog. Consider first how an analogy might be initially noticed. It is well established that human memory search is guided by semantic retrieval cues. We might suppose, then, that any semantic aspect of the novel problem could potentially provide a link to a relevant analog. There will be many potential cues to retrieve a very similar problem from the same semantic domain; for example, the radiation problem is likely to call to mind prior knowledge about related medical procedures. But by its very nature, an analog from a disparate domain will lack such transparent resemblances. Consequently, the potential semantic links between two dissimilar analogs will simply correspond to the basis of the analogy: the identities that comprise the implicit schema embedded within each analog.

It follows that if an appropriate schema has not been at least partially abstracted, it will be relatively difficult to retrieve a prior analog when given the target problem, because it is the schema that affords potential retrieval cues. Tversky's (1977) analysis of similarity implies that an analog will be more similar to its schema than to another analog, because the schema contains all the aspects common to the two analogs (mapped identities) and none of the differences between them. An independent schema will therefore facilitate the retrieval and noticing of an analogy.

In addition, it should be easier to apply a schema than an analog. An explicit problem schema will make salient those causal aspects of a situation that should trigger a particular plan of action. When two analogs are drawn from disparate domains, the inference processes underlying the mapping process will be difficult to execute; if the optimal mapping is therefore not found, the analogist may fail to generate the corresponding solution to the target problem. In general, mapping an analog to a schema will be simpler than mapping one analog with another, because in the former case it will only be necessary to map identities, rather than both identities and differences. We therefore predict that factors favoring schema induction will facilitate generation of the intended solution even if a "teacher" is available to call the person's attention to the relevant analogy.

\section{OVERVIEW OF EXPERIMENTS}

\section{Methodological Issues}

The basic procedure used to separate spontaneous noticing of an analogy from its application after a hint was adapted from Gick and Holyoak (1980) and was used in all of the present experiments. This 
procedure has three steps: (1) subjects process a story analog in the guise of a task such as story comprehension; (2) they then attempt to solve the radiation problem without any explicit hint to use the prior story; and (3) they are explicitly asked to propose a solution suggested by the story. The percentage of subjects producing the analogous solution to the target problem on their first pass provides a measure of spontaneous noticing and application; the total percentage of subjects producing the solution on either pass provides a measure of potential application given that the analogy has been noticed. The difference between these two percentages is a measure of the importance of having a "teacher" to point out the relevance of the prior analog.

This procedure is particularly sensitive to the effect of a hint, because each subject attempts to solve the problem both before and after receiving the hint. However, it also has limitations. The total percentage of subjects producing the analogous solution is not as pure a measure of potential application as would be provided by a separate group of subjects who were given a hint on their first encounter with the radiation problem. Subjects who do not immediately notice the relevance of the prior analog may tend to forget it during their initial attempts to solve the target problem. In addition, their initial attempts may produce a "set" effect that interferes with later application of the analogy. Our within-subject procedure may thus make it difficult for a condition that produces a relatively low rate of initial noticing to later "catch-up" and achieve an equal rate of total application. We should note, however, that Gick and Holyoak (1980) used both between-subject and within-subject procedures in different experiments and obtained essentially the same estimates of the percentage of subjects who could apply the analogy (compare their Experiments II and $\mathrm{V}$ ). There is therefore reason to think the potential bias associated with a within-subject procedure is not a serious one, at least for the radiation problem; nevertheless, it necessitates a degree of caution in interpreting certain of our results.

We should also note that the present study does not include any control groups that attempted to solve the radiation problem without receiving a story analog. Gick and Holyoak (1980) tested a variety of such control groups, which received either an irrelevant story that was not analogous to the radiation problem or no story at all; no more than $10 \%$ of such subjects ever produced the convergence solution. Because the present experiments used subjects drawn from essentially the same subject population, we assumed a base rate of $10 \%$ for producing the critical solution without an analogy.

The experimental manipulations used in the present study involve variations in the processing of the prior analog, rather than of the target problem. The target problem is explicitly presented as a "problem"; the 
subject can try out alternative representations of it in the course of the solution attempt. The analogous story, however, has been encoded in memory prior to presentation of the target problem. It is presented as a "story" rather than as a "problem"; subjects are never given any impetus to search for alternative representations of it, nor are they led to expect the story to later serve as the basis for a potential analogy. More generally, the analogist is free to mentally transform the representation of a situation currently being considered; but the memory representations of potential analogs will presumably have been "fixed" at the times of their original encodings and cannot be processed further until they have been retrieved and their pertinence noticed. The later accessibility of an analog should therefore be especially dependent on the nature of its initial encoding. Accordingly, all of the present experiments involve manipulations of the encodings of story analogs that are presented prior to the target problem.

\section{Manipulations of Schema Induction}

All of the present experiments investigated the effects of manipulations that might influence the induction of a problem schema. One major manipulation, which serves to organize the experiments, follows directly from our theoretical analysis. This basic variation is extremely simple-we presented subjects with either one prior story analog or else two. Our earlier analysis of schema induction suggests that this distinction is potentially crucial. A schema can be formed by abstracting the mapped identities common to two analogs. Such a mechanism clearly hinges on the provision of $t w o$ prior analogs, which can be mapped together to induce the schema. Indeed, the schema is defined by the correspondences between two analogs. Accordingly, provision of two analogs should foster induction of an independent schema, and hence facilitate subsequent transfer.

Nevertheless, the schema will be implicit within the representation of an individual analog. It is therefore conceivable that a schema could be abstracted in whole or in part from a single example. What would be required is some mechanism, other than mapping with a further example, which could make the schema explicit. Part I of the empirical section below includes three experiments that explored encoding manipulations that might promote schema induction from a single analog. The manipulations used were summarization of the prior analog (as opposed to rote recall), provision of a verbal statement of the implicit schema as part of the story, and provision of a diagrammatic representation of the schema. Part II includes three further experiments in which two prior analogs were presented. Two of the latter experiments also investigated the effects of provision of a verbal or diagrammatic representation of the schema. 


\section{PART I: REASONING FROM A SINGLE ANALOG}

\section{Experiment 1}

As noted above, Gick and Holyoak (1980) presented an initial story analogy to subjects in the guise of a recall experiment. It could be argued that the type of encoding encouraged by recall instructions may be particularly ill-suited for producing subsequent transfer to an analogous problem. Because the instructions emphasized verbatim recall, subjects may have refrained from doing any kind of abstraction that might have highlighted the convergence schema implicit in the story. Perhaps subjects would be more likely to abstract the underlying solution principle if they were required to summarize the story, rather than to recall it. The notion that summarization might foster schema abstraction acquires a degree of plausibility from evidence that summaries, as compared with recall protocols, typically include a proportionately greater number of propositions drawn from higher levels of a representational hierarchy (Thorndyke, 1977; Kintsch \& van Dijk, 1978).

Gick (Note 1) compared transfer from a story to the radiation problem when the story was presented in the context of either a recall or summarization task. She found no firm evidence that summarization instructions facilitated either abstraction of a convergence schema or subsequent transfer. Experiment 1 investigated this same issue using a different transfer problem (since the exclusive use of the radiation problem is an obvious limitation of our previous research).

Method. In choosing a new target problem we followed a conservative tack, selecting a problem that shared many of the major properties of the radiation problem. In particular, the new problem was also spatial in its nature, did not tax working memory, and allowed a varicty of potential solution plans. Our choice was Maier's $(1930,1931)$ "cord problem":

Suppose you are in a room, where two cords are hung from the ceiling. The two cords are of such a length that when you hold one cord in either hand, you cannot reach the other. Your task is to tie the ends of these cords together. To help you in this task, you may use any of the objects listed below, which are also in this room: poles, clamps, pliers, extension cords, tables, chairs.

Maier distinguished among several types of solutions to this problem, of which he was mainly interested in the "pendulum" solution. This is to tie a weight to one cord and swing it so it becomes a pendulum. The other cord can then be brought toward the center, the swinging cord can be caught as it approaches the midpoint, and then the two cords can be tied together. Maier found that $39 \%$ of his subjects produced the pendulum solution without any hints from the experimenter. This suggests that in the absence of an analogy, the pendulum solution to the cord problem is easier to discover than is the convergence solution to the radiation problem.

To provide a potential analogy, we wrote a story called "The Birthday Party" (see Appendix I), in which two ribbons are tied together after a pair of scissors is used to turn one of them into a pendulum. The story was embellished with various details more or less irrelevant to its problem-solving component. If summarization instructions lead subjects to focus on the problem-solving aspects of the story, the presence of extra details should make 
it more likely that summarization instructions will improve transfer performance relative to recall instructions.

The experiment was administered in booklet form to high school classes (grades 10 to 12) in Bloomfield, Michigan. Subjects were divided into three conditions: analog recall, analog summary, and control summary. The analog conditions either recalled or summarized the story analog prior to attempting the cord problem. The control-summary condition served to establish the base rate for production of the pendulum solution in the absence of an analogy. Subjects in the latter condition received a story that was not analogous to the cord problem ("The Wine Merchants"; see Appendix IV in Gick \& Holyoak, 1980); in other respects they received the same treatment as did those in the analog-summary condition.

Subjects were told they were participating in a two-part experiment, involving story comprehension and problem solving. Before distribution of the stories, subjects were told either that they would have to summarize or else recall the story latcr, so they should pay attention to the major points of the story (summary conditions), or else learn it in as much detail as possible (analog-recall condition). Following the 3-min period during which they studied the story, they were given detailed instructions about how to either summarize or recall it. After the protocols were written, the cord problem was distributed and subjects were required to produce as many solutions as possible. A questionnaire that queried subjects about their noticing of the story's relevance, the solution that was suggested by the story, and their prior familiarity with this solution was then distributed. Sixty-nine subjects served in the experiment: 25 in the analog-recall condition, 24 in the analog-summary condition, and 20 in the control-summary condition.

Results and Discussion. Table 2 presents the percentage of subjects in each condition who produced the pendulum solution to the cord problem before the hint, after the hint, and in total. Maximum likelihood chi squares $\left(G^{2}\right)$ were calculated for the frequency data (Bishop, Fienberg, \& Holland, 1975; Hays, 1973, p. 737, Eq. 17.8.2). The facilitative effect of a prior story analog was at least as striking as it had been in our earlier studies using the radiation problem. Combining the two analog conditions, subjects who received the analogous story produced more pendulum solutions prior to the hint than did those who received the control story $(71$ vs $20 \%), G^{2}(1)=15.8, p<.001$. The advantage of the analog conditions was maintained in a comparison of total solution frequencies (98 vs $30 \%), G^{2}(1)=30.1, p<.001$. However, the two analog conditions, recall and summary, did not differ significantly either in solutions prior to

TABLE 2

Percentage of Subjects Producing Pendulum Solution

to Cord Problem (Experiment 1)

\begin{tabular}{llccc}
\hline & $\begin{array}{c}\text { Before } \\
\text { hint }\end{array}$ & $\begin{array}{c}\text { After } \\
\text { hint }\end{array}$ & Total & $N$ \\
\hline Analog recall & $68(17)$ & $29(7)$ & $96(24)$ & 25 \\
Analog summary & $25(18)$ & $25(6)$ & $100(24)$ & 24 \\
Control summary & $20(4)$ & $10(2)$ & $30(6)$ & 20 \\
\hline
\end{tabular}

Note. Frequencies are given in parentheses. 
the hint ( 68 vs $75 \%$ ), $G^{2}(1)<1$, or in total solution frequencies ( 96 vs $100 \%)$.

The results of Experiment 1 thus yielded no evidence that summarization of the analog facilitates either noticing or applying an analogy more than does recall. While the lack of a difference in total solution frequencies could obviously be attributable to a ceiling effect, the absence of a reliable summarization advantage in initial noticing cannot. As in the comparable experiment using the radiation problem (Gick, Note 1), scrutiny of the story protocols revealed few differences between summaries and recall protocols, except that the summaries were shorter. Neither experiment yiclded any clear indication that instructions to summarize are more likely to foster either abstraction of an explicit problem schema or subsequent analogical transfer. Encoding for recall may often involve a degree of abstraction similar to that associated with encoding for summarization, particularly when study time is limited, as in the present experiment. For example, the Kintsch and van Dijk (1978) model suggests that multiple levels of macrostructure are derived in either case; summaries may be briefer than recall protocols due to differences in output processes, rather than to differences in the internal representations of the story.

The "gap" between initial noticing and eventual application of the analogy was much smaller in the present experiment with the cord problem than in prior experiments with the radiation problem. Because the experimental situations differed in many ways (e.g., the cord problem is somewhat easier to solve in the absence of an analogy), strong conclusions are unwarranted. But while we do not have a formal metric of analogical similarity with which to compare such diverse analogies as the military and radiation problems versus the ribbon and cord problems, it is intuitively compelling that the analog is semantically closer to the target problem in the latter case. For example, ribbons and cords are much more similar than are armies and rays; both pendulum solutions accomplish a goal of "tying," while the analogous convergence solutions serve either to "capture" or to "destroy." In terms of our earlier theoretical analysis, the former mapping seems to be characterized by a greater degree of identity relative to difference. The present results are thus consistent with our prediction that more similar analogs will have a greater number of potential retrieval cues to link them, so that the prior analog will more often be noticed without an explicit hint.

\section{Experiment 2}

The failure of summarization instructions to foster abstraction of a problem schema from a story analog is not surprising, given the nondirec- 
tive nature of the manipulation. Experiment 2 introduced what would seem to be a more direct approach: the story analog was augmented by an explicit verbal statement of the principle underlying the implicit problem schema. Kohler (1940) found that explaining the abstract principle upon which the solution to an algebra problem depended sometimes facilitated analogical transfer more than did provision of the initial example alone. There was therefore some reason to expect the addition of a verbal principle to be of benefit in our paradigm.

Method. The basic story analog used in Experiment 2 was "The General" (see Appendix II), with the radiation problem as the transfer task. Subjects served in one of three conditions, which differed only in the material initially studied. Those in the analog-only condition read "The General"; those in the analog-plus-principle condition read the same story, except that the following sentence was added as a final paragraph: "The general attributed his success to an important principle: If you need a large force to accomplish some purpose, but are prevented from applying such a force directly, many smaller forces applied simultaneously from different directions may work just as well." This verbal principle was meant to be an explicit statement of the essential aspects of the schema for convergence problems. Finally, subjects in the principle-alone condition did not receive the story, but only the bare statement of the principle ("If you need..."). If the verbal statement can effectively convey the convergence schema to subjects, even without an accompanying specific example, then subjects in the latter condition should produce the convergence solution to the radiation problem more frequently than the base rate.

Subjects were tested in small groups. Those in the analog-only and analog-plus-principle conditions were told they were first to learn a story; they then were allowed 3 min to read the story, after which they were asked to recall it. Subjects in the principle-only condition were given a slightly different task. They were told the first part of the experiment involved "selecting statements to use in a study of comprehension" and that they were to first read a statement and prepare to paraphrase it. After studying the statement for $3 \mathrm{~min}$, they were asked to paraphrase it and to rate its plausibility and comprehensibility. After their initial task was completed, subjects in all conditions attempted to solve the radiation problem in the usual two-pass manner: first without a hint to use the prior story or statement, and then with such a hint.

Eighty-eight subjects participated in the experiment, distributed approximately equally across the three conditions.

Results and Discussion. Table 3 presents the percentage of subjects in each of the three conditions who produced the convergence solution before the hint, after it, and in total. The results were straightforward: solution frequencies did not differ significantly among the conditions,

TABLE 3

Percentage of Subjects Producing Convergence Solution (Experiment 2)

\begin{tabular}{lllll}
\hline & $\begin{array}{c}\text { Before } \\
\text { hint }\end{array}$ & $\begin{array}{c}\text { After } \\
\text { hint }\end{array}$ & Total & $N$ \\
\hline Analog plus principle & $32(10)$ & $48(15)$ & $80(25)$ & 31 \\
Analog only & $29(8)$ & $50(14)$ & $79(22)$ & 28 \\
Principle only & $28(8)$ & $38(11)$ & $66(19)$ & 29 \\
\hline
\end{tabular}

Note. Frequencies are given in parentheses. 
either before the hint, $G^{2}(2)<1$, or in total, $G^{2}(2)=2.05, p=.36$. In particular, the performance of subjects in the analog-plus-principle condition was virtually identical to that of those in the analog-only condition: about $30 \%$ produced the convergence solution without a hint, and about $80 \%$ produced it in total. These figures are comparable to those obtained in previous experiments that used the military story and radiation problem. Experiment 2 thus yielded no support for the hypothesis that augmenting the story analog with a verbal principle would increase analogical transfer.

This does not mean, however, that the verbal principle was totally incffective, because a substantial proportion of subjects in the principleonly condition also produced the convergence solution. In all three conditions (considered separately) the $95 \%$ confidence limits for the percentage of such successful subjects excluded the base rate of $10 \%$, even prior to the hint. It is therefore clear that the information in the verbal statement was often sufficient to allow generation of the convergence solution to the transfer problem; however, once the subject received a specific story analog, addition of the abstract principle conveyed no further benefit.

It therefore seems that the verbal principle did not foster abstraction of the convergence schema from the analog. One possibility is that subjects who received the story simply ignored the concluding statement. However, inspection of the recall protocols for the analog-plus-principle subjects suggested otherwise. All but three of the 31 subjects included at least one aspect of the abstract statement of the convergence principle in their recall of the story. It is possible, however, that subjects did not attend carefully to the relationship between the final statement and the concrete instantiation of a convergence solution embedded in the story. That is, they may not have mapped the story and the statement to abstract the convergence schema implicit in the story itself. This seems particularly plausible given that they were simply asked to learn the story and did not expect to have to apply the information in a subsequent problem-solving task. The present findings therefore should not be taken as evidence that a verbal principle can never aid in clarifying an example. Augmenting an example with a principle may be beneficial when the information is presented in an explicit problem-solving context (Kohler, 1940), rather than in an incidental task. However, our results indicate that simply adding a principle to a specific analog by no means guarantees that an abstract schema will be abstracted.

\section{Experiment 3}

Although the problems used in our experiments have always been stated verbally, they are fundamentally spatial in nature. Perhaps an abstract principle would better serve to highlight the convergence schema 
implicit in a single analog if the principle were presented in a spatial rather than a verbal mode. To test this possibility, Experiment 3 used a design analogous to that of Experiment 2, but with visual diagrams instead of a verbal statement of the principle.

Method. Subjects again served in one of three conditions. Those in the analog-only condition read the same military story as had been used in the comparable condition of Experiment 2. Subjects in the analog-plus-diagrams condition also read the same story, but modified slightly to refer to a pair of accompanying diagrams, depicted in Fig. 1. Diagram A, a single large arrow, represents the desirable but blocked plan of sending a large force from a single direction. The story referred to this diagram as an illustration of the general's initial plan "to launch a full-scale direct attack." Diagram B, several smaller converging arrows, represents the alternative, successful plan of sending small forces from multiple directions. The story referred to the latter diagram as an illustration of the general's solution in a sentence inserted immediately before the last sentence of the story (see Appendix II). These simple arrow diagrams were intended to represent the convergence principle at an abstract level, not necessarily tied to the story's specific military context.

Subjects in both of the above conditions were told to pay attention to the major points of the story during their 3-min study period and that they would later have to summarize it. Those in the analog-plus-diagrams condition were told in addition to pay attention to the diagrams and to incorporate them into their summaries. After the study period subjects in both analog conditions were asked to write a summary and to rate the story's plausibility and comprehensibility. Subjects in a third condition, diagrams only, received the diagrams without any accompanying story. They were told that the first part of the experiment involved pattern recognition; they were to study the diagrams for $3 \mathrm{~min}$ so that they could later reproduce them. These subjects then drew the diagrams from memory and rated their perceptual complexity and the difficulty of reproducing them.

After completing their initial task, all subjects received the radiation problem and final questionnaire in the usual two-pass manner. The final questionnaire for the diagrams-only condition asked subjects to give a solution suggested by the prior diagrams. Sixty-six subjects served in the experiment: 26 in the analog-plus diagrams condition, 25 in the analogonly condition, and 15 in the diagrams-only condition.

Results and Discussion. Table 4 presents the percentage of subjects in each condition who produced the convergence solution to the radiation problem. There was no indication that spatial diagrams, any more than the verbal statement used in Experiment 2, constituted an effective addition to the basic story analog. The percentage of subjects producing the convergence solution prior to the hint was actually lower in the analog-plusdiagrams condition than in the analog-only condition, although not signifi-

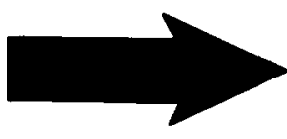

A

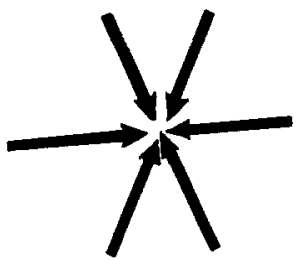

8

Fig. 1. The two diagrams used in Experiments 3 and 6 to illustrate the principle underlying the convergence solution. 
TABLE 4

Percentage of Subjects Producing Convergence Solution

(Experiment 3)

\begin{tabular}{lcccc}
\hline & $\begin{array}{c}\text { Before } \\
\text { hint }\end{array}$ & $\begin{array}{c}\text { After } \\
\text { hint }\end{array}$ & Total & $N$ \\
\hline Analog plus diagrams & $23(6)$ & $58(15)$ & $81(21)$ & 26 \\
Analog only & $40(10)$ & $36(9)$ & $76(19)$ & 25 \\
Diagrams only & $7(1)$ & $60(9)$ & $67(10)$ & 15 \\
\hline
\end{tabular}

Note. Frequencies are given in parentheses.

cantly so, $G^{2}(1)=1.71, p=.19$. The total solution frequencies in both conditions were very similar (approximately 80\%), $G^{2}(1)<1$.

Although both analog conditions yielded results much like those in previous comparable experiments, the diagrams-only condition provided an interesting contrast. Combining the two analog conditions, $31 \%$ of their subjects produced the convergence solution without the hint, as compared to only one of the 15 diagrams-only subjects (7\%). The latter subject reported solving the radiation problem without thinking of the diagrams. However, nine additional diagrams-only subjects generated the target solution once the hint was given; the total for that condition, $67 \%$, was not significantly less than the figure of $78 \%$ obtained for the analog conditions, $G^{2}(1)<1$. The diagrams-only condition thus yielded the most striking discrepancy between initial noticing and eventual application we have yet observed: information in memory, sufficient to trigger the convergence solution for most subjects, was literally never spontaneously noticed.

This result in fact supports our semantic retrieval-cue analysis of noticing analogies. When the diagrams were presented alone in the context of a "pattern recognition" task, it is unlikely that subjects assigned any semantic interpretation to them, and surely none even approximating the convergence schema. As a result, no semantic retrieval cues were subsequently available to link the radiation problem with the diagrams. But once subjects had been explicitly told to consider the prior diagrams, they were able to interpret them by means of a mapping process. For example, they may have noticed the abstract similarity of the large unidirectional arrow to the desirable but blocked possibility of directing high-intensity rays at the tumor. Once the initial arrow had been interpreted as a representation of the intensity and direction of rays, the relationship between the two diagrams could be construed as a transformation of one large unidirectional ray into several small converging ones, thus illustrating the convergence solution. Such a chain of reasoning might be termed "analogical bootstrapping." The antecedent conditions given in the statement of the transfer problem are first used to interpret the 
"antecedent" diagram; once the components of the first diagram have been interpreted by constructing an initial mapping, the "consequence" represented by the second diagram can be decoded.

But if the diagrams can indeed be interpreted as an abstract analog to the radiation problem, why did they not facilitate analogical transfer when presented in the context of the military story? They certainly were not ignored; every subject reproduced them accurately after the study period. One might have expected the diagrams to highlight the abstract convergence schema implicit in the story. But as was the case with the verbal statement used in Experiment 2, scrutiny of subjects' story protocols indicated that nothing of the sort actually occurred. Except for references to the diagrams, there were no obvious differences between the summaries written by subjects in the analog-plus-diagrams conditions and those written by subjects in the analog-only condition. In neither case was there anything in the protocols to suggest a more abstract interpretation of the general's solution strategy, which might apply to disparate problems. In fact, there were signs that rather than the diagrams providing a more abstract interpretation of the story, the story provided a more specific interpretation of the diagrams. Two subjects stated that the general divided his men into six groups, which were dispatched to six roads, "as illustrated in the diagram." The story does not say how many roads were used, but the second diagram indeed shows six arrows. It seems that in the story context the diagrams were simply taken to represent roads, rather than abstract directions of force. Given that the diagrams did not encourage abstraction of the convergence schema from the story analogy, their failure to increase analogical transfer is entirely in accord with our earlier analysis.

\section{PART II: FORMATION AND USE OF SCHEMAS}

We have examined three manipulations-summarization instructions, addition of a verbal statement of the underlying principle, and addition of a diagrammatic representation of it-that were designed to promote spontaneous transfer by making the schema latent in the story analog more explicit. These all failed to facilitate transfer from a single analog. Furthermore, none of these manipulations appeared to foster abstraction of a general problem schema from one example, even though the verbal statement amounted to an explicit statement of such a schema, and both the verbal statement and the diagram produced transfer when presented alone (although in the latter case only with a hint). A common limitation of all these procedures is that they do not allow induction of a schema by mapping, since only one example was provided. The experiments in Part II investigated the induction of a problem schema from multiple analogs. 


\section{Experiment 4}

Experiment 4 explicitly compared the effectiveness of two vs one prior analogs in producing analogical transfer. In addition, the experimental procedure gave subjects an opportunity to write down a description that could approximate the convergence schema. The quality of their schema descriptions could then be related to their subsequent performance in solving the transfer problem.

As well as simply providing a second analog, we varied whether the two story analogs were drawn from similar or dissimilar domains (where the analog domains were always dissimilar to the medical domain of the radiation problem). There is reason to expect the effect of analog similarity to be complex. Studies of perceptual category learning have often found that training with relatively diverse examples leads to superior transfer performance (Posner \& Keele, 1968; Fried \& Holyoak, Note 3); comparable effects have been demonstrated with artificial semantic concepts (Nitsch, Note 4). On the other hand, perceptual categories are learned more slowly when the training exemplars are highly variable (Fried \& Holyoak, Note 3 ); and when the number of initial exemplars is very small, high variability can actually impair transfer performance (Peterson, Meagher, Chait, \& Gillie, 1973; Homa \& Vosburgh, 1976).

The general picture appears to be that dissimilar training examples make it more difficult to lcarn a concept, but allow it to be used more flexibly once acquired. A comparable pattern might be expected in the present paradigm. In terms of our earlier analysis, the optimal schema for a set of analogs will be a representation that captures the essential relations relevant to the solution, while excluding irrelevant domain-specific details. The schema that can be abstracted from two analogs will depend on the set of mapped identities they share. Because the optimal convergence schema is quite abstract, it would seem that the mapped identities linking two relatively dissimilar analogs would more likely correspond to an optimal schema (because they are similar only at an abstract level). A schema induced from two similar examples would often include information too specific to apply to a disparate transfer problem. However, the mapping process may be substantially more difficult to accomplish if the analogs are dissimilar, because the differences will be many and the identities few and abstract. In contrast, it will be relatively easy to map two similar analogs, which will afford salient and specific points of correspondence. Thus, while a mapping between dissimilar analogs may potentially yield relatively optimal schemas, the actual schema that results may fall short of that which emerges from a mapping between two similar analogs.

Method. Four story analogs to the radiation problem were used (see Appendix II). One was "The General" (by now a veteran of many campaigns in our previous experiments); the 
other three were written for this experiment. "The Commander," a story involving an attack on an island using multiple bridges, provided a second military analog. The other pair of stories shared a fire-fighting theme. "Red Adair" had the resourceful hero extinguish an oil-well fire by using multiple hoses; "The Fire Chief" accomplished a similar feat with the aid of a bucket brigade.

All subjects initially read two stories. Subjects were divided into three basic groups, each of which received a different type of story pair. Those in the two-similar-analogs condition received either the two military stories or the two fire-fighting stories, while those in the two-dissimilar-analogs condition received one of each. Subjects in the analog-plus-control condition received just one story analog, plus a disanalogous control story ("The Identical Twins") that had proved useless as a prompt for the convergence solution in earlier experiments (Gick \& Holyoak, 1980). Across different subjects, all four story analogs were used about equally often within each of these three conditions, and the order of the stories was counterbalanced.

Subjects were told that the first part of the experiment involved screening some stories for use in a study of story comprehension. They were asked to read each story in the given order and to write brief summaries of each, with the stories still available for reference. Subjects were allowed to keep the stories while answering questions about them in order to facilitate comparison of one to the other. The subjects also rated the comprehensibility of each story. Then, in what may be the most critical aspect of the procedure, subjects were asked to describe in writing, as clearly as possible, ways in which the stories were similar. This task was intended to elicit a mapping between one story and the other, and hence to potentially lead to abstraction of a convergence schema. Furthermore, the written descriptions could later be scored for presence and quality of the schema. Finally, subjects were asked to rate the similarity of the two stories on a 7-point scale. The radiation problem was then presented in the usual two-pass manner, except that the final questionnaire asked subjects to write down a solution suggested by either one or both of the prior stories.

A total of 98 subjects were tested: 28 in the two-similar-analogs condition, 23 in the two-dissimilar-analogs condition, and 47 in the analog-plus-control condition. Subjects in the latter condition were divided into four approximately equal subgroups, each of which read a different story analog, plus the irrelevant story.

Results and Discussion. Table 5 presents the percentage of subjects in each condition who produced the convergence solution prior to the hint, after it, and in total. While there was a small trend toward a greater frequency of unprompted solutions when the two prior analogs were dissimilar rather than similar (52 vs $39 \%$ ), this difference did not approach significance, $G^{2}(1)<1$. Because these two conditions that received two analogs also did not differ in total solution frequencies, they were combined in order to make a comparison with the one-analog-plus-control

TABLE 5

Percentage of Subjects Producing Convergence Solution

(Experiment 4)

\begin{tabular}{lcccc}
\hline & $\begin{array}{c}\text { Before } \\
\text { hint }\end{array}$ & $\begin{array}{c}\text { After } \\
\text { hint }\end{array}$ & Total & $N$ \\
\hline Two similar analogs & $39(11)$ & $39(11)$ & $79(22)$ & 28 \\
Two dissimilar analogs & $52(12)$ & $31(7)$ & $83(19)$ & 23 \\
One analog plus control & $21(10)$ & $32(15)$ & $53(25)$ & 47 \\
\hline
\end{tabular}

Note. Frequencies are given in parentheses. 
condition. This comparison yielded clear differences. Forty-five percent of subjects in the two-analog conditions generated the convergence solution prior to the hint, as opposed to only $21 \%$ of subjects in the one-analog condition, $G^{2}(1)=6.35, p<.01$. A comparable advantage for the twoanalog conditions was obtained when total solution frequencies were compared ( 80 vs $53 \%), G^{2}(1)=8.36, p<.003$. It was clearly the case that two analogs were better than one.

We next sought to assess the relationship between quality of the schemas induced by subjects, as evidenced by their descriptions of the similarities between the two stories, and analogical transfer. This analysis was performed only for the two-analog conditions. The descriptions were categorized into three levels of schema quality. In order for a description to qualify as a "good" schema, the basic idea of having forces converge from different directions had to be present either explicitly or as an inference. (The idea of using different directions was inferred if the subject mentioned the simultaneous use of multiple forces.) In addition, at least one other major aspect of the analogy had to be expressed: either the use of multiple small forces or the parallels in the initial problem situations (e.g., centrally located targets). An example of a good schema is: "Both stories used the same concept to solve a problem, which was to use many small forces applied together to add up to one large force necessary to destroy the object." An "intermediate" schema contained only one of these major features; e.g., "In both cases many small forces were used." "Poor" schemas contained none of the basic aspects of the convergence principle. They usually either alluded to a similarity between the solutions that was abstract to the point of vacuity (e.g., "In both stories a problem was solved using logical means") or did not focus on the problem-solving aspects of the stories at all (e.g., "In both stories a hero was rewarded for his efforts"). The descriptions were evaluated by two independent judges, and disagreements, which were infrequent, were resolved by discussion.

The schema analysis revealed no significant differences as a function of similarity of the two analogs; nor did the relationship between schema quality and transfer performance vary reliably across the two conditions (although the cell frequencies were too low for the latter null result to be taken at all seriously). Combining both conditions, $21 \%$ of subjects produced good schemas, $20 \%$ produced intermediate schemas, and the majority, $59 \%$, produced poor schemas. The question of central interest is whether schema quality was at all predictive of success in generating the convergence solution to the radiation problem. As the data in Table 6 indicate, such a relationship is strikingly evident. The cell frequencies presented in Table 6 differ reliably, $G^{2}(4)=15.8, p<.005$, and the pattern of difference is quite simple: the better the schema, the more successful was transfer performance. Thus, fully $91 \%$ of subjects scored as having good schemas generated the convergence solution without a hint; none 
TABLE 6

Percentage of Subjects Producing Convergence Solution at Each Stage as a Function of Schema Quality (Experiment 4)

\begin{tabular}{lcccc}
\hline & \multicolumn{3}{c}{ Timing of convergence solution } & \\
\cline { 2 - 4 } Schema Quality & $\begin{array}{c}\text { Before } \\
\text { hint }\end{array}$ & $\begin{array}{c}\text { After } \\
\text { hint }\end{array}$ & $\begin{array}{c}\text { Not } \\
\text { produced }\end{array}$ & $N$ \\
\hline Good & $91(10)$ & $9(1)$ & $0(0)$ & 11 \\
Intermediate & $40(4)$ & $50(5)$ & $10(1)$ & 10 \\
Poor & $30(9)$ & $40(12)$ & $30(9)$ & 30 \\
\hline
\end{tabular}

Note. Frequencies are given in parentheses.

failed to produce it eventually. Forty percent of subjects with intermediate schemas produced the target solution without a hint, and $90 \%$ produced it in total. The comparable figures for those with poor schemas were $30 \%$ unprompted and $70 \%$ in total. To state the results in different terms, $90 \%$ of the subjects who failed to ever produce the convergence solution were scored as having poor schemas.

One must be cautious in suggesting a causal basis for this strong relationship between schema quality and transfer performance; it may be that people who are skillful problem solvers write good schemas and also apply analogies well. However, our subsequent experiments will lend a degree of support for a causal interpretation. The observed pattern is certainly consistent with the view that the task of evaluating story similarities invokes a mapping process which may lead to the incidental abstraction of a problem schema; the more closely the induced schema approximates the optimal convergence schema, the more likely the person is to notice its relevance to the subsequent transfer problem and to be able to derive the appropriate solution.

The schema analysis also suggests explanations of what would otherwise seem to be puzzling aspects of the performance levels observed in the present transfer task. First, the two-analog conditions yielded solution frequencies of $45 \%$ without a hint and $80 \%$ in total. At least the latter figure is not remarkably different from figures obtained in previous experiments with a single prior analog, which might seem to contradict our prediction that reasoning from a schema will be more effective than reasoning from an analog. However, the schema analysis revealed that virtually $60 \%$ of the subjects in the two-analog conditions produced poor schemas, which is to say no part at all of the convergence schema. Those subjects who wrote intermediate or good schemas indeed outperformed any of our previous one-analog conditions.

A second and related puzzle is why the present one-analog condition did so poorly: $21 \%$ solutions without a hint, $53 \%$ in total, as opposed to the usual figures of roughly 30 and $75 \%$. Although three of the four story 
analogies had not been used in previous experiments, the new stories were no less effective than the old one. However, note that the present one-analog-plus-control condition actually involved two stories, only one of which was an analogy to the radiation problem; furthermore, subjects in this condition also described the similarities between the two stories, just as did those in the two-analog conditions. The procedure thus encouraged analog-plus-control subjects to also abstract some sort of schema linking their two stories. But because the two stories were totally disanalogous, except that both described problem situations, the resulting schemas were invariably poor-indeed, very poor-approximations to the convergence schema. For these subjects, the induced schema may have actually interfered with their ability to later retrieve and apply the actual analog during the transfer task.

\section{Experiment 5}

Experiment 4 yielded clear evidence that a problem schema can be induced by mapping two analogs and that the quality of that schema predicts subsequent transfer performance. However, only about $40 \%$ of the subjects described the similarities between the two analogs in terms that approximated the convergence schema. Our analysis predicts that any manipulation that can facilitate schema formation will boost analogical transfer. Once the subject has been given two analogs, the mapping process should be aided by any device that calls attention to the critical correspondences. We have already introduced two such devices in earlier experiments: a verbal statement of the convergence principle and a diagrammatic representation of it. Neither manipulation facilitated schema abstraction from a single analog. However, these negative results may simply reflect the fact that a mapping process requires two analogs in order to operate at all. Once this prerequisite is met, highlighting devices should have a positive effect. Experiment 5 therefore examined the effect of adding the verbal statement, used previously in Experiment 2, to two prior analogs.

Method. To simplify the experimental design only one pair of dissimilar story analogs were used: "The General" and "The Fire Chief." Subjects served in one of two conditions. Those in the without-principle condition read the two stories just as they appear in Appendix II. Those in the with-principle condition read the identical stories, except that the verbal statement of the convergence principle, previously used in Experiment 2, was appended as the final paragraph of each. The statement was worded exactly as it had been in the earlier experiment (except, of course, that the corresponding sentence for "The Fire Chief' began, "The fire chief attributed his success. . "). The statement was thus designed to focus the subjects' attention on the critical aspects of the schema implicit in each of the two analogs.

The two-analog procedure of Experiment 4 was modified slightly so as to be more comparable with that of Experiment 2. Subjects were first told to study the two stories carefully for $5 \mathrm{~min}$ in preparation for answcring questions about them. The stories were then collected, and the remainder of the initial story task was done from memory. Subjects were asked to briefly summarize each story, rate the comprehensibility of each, describe as clearly as 
possible the ways in which the situations in the two stories seemed similar, and rate their overall similarity. After this initial task was completed, the radiation problem was administered in the usual two-pass manner.

Subjects were tested in several different classrooms. A total of 143 subjects were divided about evenly between the two conditions.

Results and Discussion. In sharp contrast to the comparable one-analog study (Experiment 2), addition of the verbal statement had a clear positive effect on transfer from a pair of analogs. As is shown in Table 7A, 62\% of the subjects in the with-principle condition produced the convergence solution without a hint, as compared to only $40 \%$ of those in the withoutprinciple condition, $G^{2}(1)=6.75, p<.01$. The frequency of noticing in the former condition is in fact higher than that obtained in any of our previous experiments with the radiation problem. The with-principle condition maintained a slightly smaller advantage in total solution frequency ( $82 \mathrm{vs}$ $67 \%), G^{2}(1)=4.34, p<.05$.

Subjects' similarity descriptions were scored for schema quality in essentially the same manner as in Experiment 4. A few subjects in the with-principle condition simply said that the two stories illustrated the same principle; such descriptions were counted as good schemas only if the principle was clearly stated in their summary of each story. As the data in Table 7B indicate, addition of the principle had a strong influence on schema quality, $G^{2}(2)=28.4, p<.001$. Sixty percent of subjects in the without-principle condition wrote poor schemas, just as in the comparable condition of Experiment 4 ; only $10 \%$ gave good schemas. In contrast, only $23 \%$ of those in the with-principle condition produced poor schemas, whereas $44 \%$ produced schemas scored as good.

TABLE 7

A. Percentage of Subjects Producing Convergence

Solution (Experiment 5)

\begin{tabular}{lcccc}
\hline & $\begin{array}{c}\text { Before } \\
\text { hint }\end{array}$ & $\begin{array}{c}\text { After } \\
\text { hint }\end{array}$ & Total & $N$ \\
\hline With principle & $62(45)$ & $20(15)$ & $82(60)$ & 73 \\
Without principle & $40(28)$ & $27(19)$ & $67(47)$ & 70 \\
\hline
\end{tabular}

B. Percentage of Subjects at Each Level

of Schema Quality

\begin{tabular}{llccc}
\hline & \multicolumn{3}{c}{ Schema quality } & \multirow{2}{*}{$N$} \\
\cline { 2 - 4 } & Good & Intermediate & Poor & $N$ \\
\hline With principle & $44(32)$ & $33(24)$ & $23(17)$ & 73 \\
Without principle & $10(7)$ & $30(21)$ & $60(42)$ & 70 \\
\hline
\end{tabular}

Note. Frequencies are given in parentheses. 
TABLE 8

Percentage of Subjects Producing Convergence Solution at Each Stage as a Function of Schema Quality (Experiment 5)

\begin{tabular}{lllll}
\hline & \multicolumn{2}{c}{ Timing of convergence solution } & \\
\cline { 2 - 4 } Schema Quality & $\begin{array}{c}\text { Before } \\
\text { hint }\end{array}$ & $\begin{array}{c}\text { After } \\
\text { hint }\end{array}$ & $\begin{array}{c}\text { Not } \\
\text { produced }\end{array}$ & $N$ \\
\hline With principle & & & & \\
$\quad$ Good & $75(24)$ & $19(6)$ & $6(2)$ & 32 \\
Intermediate & $58(14)$ & $29(7)$ & $13(3)$ & 24 \\
Poor & $41(7)$ & $12(2)$ & $47(8)$ & 17 \\
Without principle & & & & \\
Good & $57(4)$ & $29(2)$ & $14(1)$ & 7 \\
Intermediate & $57(12)$ & $24(5)$ & $19(4)$ & 21 \\
Pour & $29(12)$ & $29(12)$ & $43(18)$ & 42 \\
\hline
\end{tabular}

Note. Frequencies are given in parentheses.

Table 8 presents data regarding the relationship between schema quality and transfer performance. Data for the two experimental conditions were tabulated and analyzed separately. For the with-principle condition, schema quality had a strong influence on generation of the convergence solution, $G^{2}(4)=13.0, p<.02$. For example, fully $94 \%$ of the subjects who wrote good schemas eventually produced the solution, as opposed to only $53 \%$ of those who wrote poor schemas. The overall relationship between schema quality and solution generation was less robust for the without-principle condition, $G^{2}(4)=7.00, p=.14$, due to the lack of clear differences between subjects who gave good versus intermediate schemas. However, little weight can be attached to this null finding, because only seven subjects in this condition wrote good schemas. When subjects with good and intermediate schemas were combined, schema quality had a significant positive effect for the without-principle condition as well, $G^{2}(2)=6.89, p<.05$. Eighty-six percent of the subjects who gave descriptions relevant to the convergence principle eventually produced the target solution, as opposed to only $57 \%$ of those who gave poor schemas.

\section{Experiment 6}

The results of Experiment 5 again provided clear evidence of the formation and use of schemas in analogical problem solving; furthermore, they demonstrated that a verbal statement of the solution principle can aid in the induction of a schema from two analogs and substantially improve both noticing and application of the information in a subsequent transfer task. Experiment 6 was designed to seek comparable effects of a visual 
representation of the principle. The diagrams previously used in Experiment 3, where they failed to facilitate abstraction of a schema from one analog, were now presented along with two analogs. In addition, Experiment 6 obtained further evidence regarding the influence of similarity between the two prior analogs, which had yielded no clear effects in Experiment 4.

As we argued in connection with Experiment 4, there are reasons to expect variations in similarity of the analogs to have complex and sometimes offsetting effects. We would expect provision of diagrams or other devices that highlight the problem schema implicit in each analog to foster induction of the schema from either similar or dissimilar analogs, but for somewhat different reasons. The danger with similar analogs is that the mapping process will yield a set of mapped identities that includes domain-specific details irrelevant to the more general schema. The diagrams in Fig. 1 should reduce this problem, by focusing the subject's attention on those central correspondences causally related to the analogous solution plans. The difficulty raised by a pair of dissimilar analogs is essentially the converse: the analogical correspondences will be few and relatively abstract, so that the mapping process may simply fail to find them. In this case the diagrams should also prove helpful, because they will serve to isolate the essential correspondences which they abstractly represent.

Method. Subjects served in one of four basic conditions, all of which received two story analogies. The four stories used in Experiment 4 were again used in Experiment 6 . Subjects read either two similar analogs (both from either the military or the fire-fighting domain) or else two dissimilar analogs (one from each domain). The assignment of stories to conditions, and the order of the two stories in a pair, were fully counterbalanced, so that across subjects each story was used about equally often in similar and dissimilar pairs. Subjects in the without-diagrams conditions read the stories as they are presented in Appendix II. Those in the with-diagrams conditions received a separate sheet of paper with the two arrow diagrams illustrated in Fig. 1. The stories in these conditions were each modified slightly to refer to the diagrams appropriately. just as in Experiment 3.

The procedure of Experiment 6 was essentially the same as that of Experiment 5. Subjects first studied the stories for five min and then summarized them and described their similarities from memory. Subjects in the with-diagrams conditions were also asked to draw the diagrams. Following the story task the radiation problem was administered as usual. The subjects, 189 in all, were run in small groups; approximately equal numbers of subjects served in each of the four conditions.

Results and Discussion. Table 9A presents the percentage of subjects in each condition who produced the convergence solution before the hint, after it, and in total. Provision of a diagram resulted in a higher frequency of initial solutions ( 57 vs $37 \%$ ), $G^{2}(1)=7.55, p<.01$, as well as of total solutions ( 92 vs $79 \%), G^{2}(1)=6.66, p<.01$. This total solution frequency obtained when subjects received two analogs plus the diagrams, $92 \%$, is higher than that observed in any previous experiment with the radiation 
TABLE 9

A. Percentage of Subjects Producing Convergence Solution (Experiment 6)

\begin{tabular}{lcccc}
\hline & $\begin{array}{c}\text { Before } \\
\text { hint }\end{array}$ & $\begin{array}{c}\text { After } \\
\text { hint }\end{array}$ & Total & $N$ \\
\hline Similar analogs with diagrams & $61(29)$ & $33(16)$ & $94(45)$ & 48 \\
Dissimilar analogs with diagrams & $53(27)$ & $37(19)$ & $90(46)$ & 51 \\
Similar analogs without diagrams & $40(17)$ & $40(17)$ & $79(34)$ & 43 \\
Dissimilar analogs without diagrams & $34(16)$ & $45(21)$ & $79(37)$ & 47 \\
\hline
\end{tabular}

B. Percentage of Subjects at Each Level of Schema Quality

\begin{tabular}{lcccc}
\hline & \multicolumn{3}{c}{ Schema quality } & \\
\cline { 2 - 4 } & Good & Intermediate & Poor & \multirow{2}{*}{$N$} \\
\hline Similar analogs with diagrams & $44(21)$ & $29(14)$ & $27(13)$ & 48 \\
Dissimilar analogs with diagrams & $65(33)$ & $18(9)$ & $18(9)$ & 51 \\
Similar analogs without diagrams & $26(11)$ & $30(13)$ & $44(19)$ & 43 \\
Dissimilar analogs without diagrams & $30(14)$ & $36(17)$ & $34(16)$ & 47 \\
\hline
\end{tabular}

Note. Frequencies given in parentheses.

problem: nine times higher than the base rate for generation of the solution without any analog, it is clearly close to the maximum any manipulation could achieve. ${ }^{5}$ The facilitating effects of the diagrams were essentially the same for similar and dissimilar analogs; analog similarity had no significant influence on solution frequencies.

Subjects' descriptions of the similarities between the two stories were again scored for schema quality. A few subjects in the with-diagrams conditions explicitly referred to the diagrams as representing the similar aspects of the two situations. We made the assumption that such subjects understood the underlying principle, and scored them as having good schemas. (If this assumption were unduly generous, it should reduce the relationship between schema quality and transfer performance for the with-diagrams conditions.) Table 9B presents the percentages of subjects at the three levels of schema quality, for each of the four conditions. Provision of the diagrams improved the quality of the schemas derived from both similar and dissimilar analogs, $G^{2}(2)=14.4, p<.001$. Of subjects who received diagrams, $55 \%$ wrote good schemas and only $22 \%$ wrote poor ones; of those who did not receive diagrams, only $28 \%$ gave good schemas while $39 \%$ gave poor ones. Thus, once again, as in Experi-

\footnotetext{
${ }^{5}$ In an earlier experiment using one pair of dissimilar analogs (Gick, Note 1), the addition of the diagrams yielded solution frequencies of $68 \%$ without a hint and $95 \%$ in total $(N=37)$. The results of this study were replicated in all comparable respects by those of Experiment 6 .
} 
ment 5, a manipulation that facilitated schema induction also increased the degree of analogical transfer.

When no diagrams were given, analog similarity did not influence schema quality, $G^{2}(2)<1$. However, when the diagrams were presented, there was a tendency for dissimilar analogs to yield a higher frequency of good schemas than was the case for similar analogs (65 vs $44 \%$ ). This trend was in fact significant, $G^{2}(1)=4.41, p<.05$; it provides a hint that a mapping between relatively disparate analogs, when guided by a device that highlights the underlying solution principle, may be more likely to generate the optimal schema. However, as noted above, this increase in the proportion of good schemas did not translate into superior transfer performance for subjects given dissimilar analogs. The data reported below will shed light on this apparent discrepancy.

The data in Table 10 convey the relationship between schema quality and success in generating the analogous solution to the transfer problem. Because this pattern did not differ significantly as a function of whether diagrams were provided, the data have been collapsed across that variable. When the prior analogs were dissimilar to each other, the usual positive relationship was found: the better the schema, the more likely the convergence solution was to be produced, especially prior to the hint, $G^{2}(4)=21.5, p<.001$. But somewhat surprisingly, the relationship was far less strong when the prior analogs were similar, $G^{2}(4)=5.16, p=.27$. Some degree of positive relationship was still apparent, which was significant when the most extreme cells were compared (the frequencies with which solutions were produced before the hint vs not at all, for subjects with good vs poor schemas), $G^{2}(1)=3.98, p<.05$.

TABLE 10

Percentage of Subjects Producing Convergence Solutions at Each Stage as a Function of Schema Quality and Similarity of the Prior Analogs (Experiment 6)

\begin{tabular}{lllll}
\hline & \multicolumn{3}{c}{ Timing of convergence solution } \\
\cline { 2 - 4 } Schema quality & $\begin{array}{c}\text { Before } \\
\text { hint }\end{array}$ & $\begin{array}{c}\text { After } \\
\text { hint }\end{array}$ & $\begin{array}{c}\text { Not } \\
\text { produced }\end{array}$ & $N$ \\
\hline Similar analogs & & & & \\
$\quad$ Good & $63(20)$ & $31(10)$ & $6(2)$ & 32 \\
Intermediate & $44(12)$ & $45(12)$ & $11(3)$ & 27 \\
Poor & $44(14)$ & $34(11)$ & $22(7)$ & 32 \\
Dissimilar analogs & & & & \\
Good & $64(30)$ & $28(13)$ & $8(4)$ & 47 \\
Intermediate & $39(10)$ & $50(13)$ & $11(3)$ & 26 \\
Poor & $12(3)$ & $56(14)$ & $32(8)$ & 25 \\
\hline
\end{tabular}

Note. Frequencies given in parentheses. 
In fact, the only substantial difference in Table 10 between the patterns obtained for similar vs dissimilar analogs involved the relative frequencies with which subjects who wrote poor schemas produced the solution without a hint (44\% for similar analogs vs $12 \%$ for dissimilar analogs). There is a sense in which our measure of schema quality establishes only a lower bound on what subjects have learned from the prior analogs; they may write down less than they know. Those who receive similar analogs may sometimes write down only similarities irrelevant to the convergence principle, and hence be scored as having poor schemas; nevertheless, they may have actually abstracted the critical correspondences to some degree. This is less likely in the case of subjects who receive dissimilar analogs; because there are few superficial resemblances to describe, those who write poor schemas have probably really missed the basic analogy. Thus, while dissimilar stories, when accompanied by the diagrams, yielded a higher frequency of good schemas, those subjects who wrote poor schemas on the basis of dissimilar analogs were less successful at transfer than were their counterparts who wrote poor schemas on the basis of similar analogs. This pattern is in accord with our hypothesis regarding analog similarity: while dissimilar analogs have greater potential to yield optimal schemas, they are also more likely to fail to produce any useful schema. At a descriptive level, this tradeoff accounts for the absence of differences in overall transfer performance as a function of similarity of the prior analogs.

\section{GENERAL DISCUSSION}

\section{Summary and Implications}

The expcriments in Part I attempted to foster the abstraction of a problem schema from a single story analog by means of summarization instructions, or else either verbal or visual statements of the underlying principle. We found no evidence that any of these devices yielded more abstract representations of the story, nor did any consistently facilitate analogical transfer. In contrast, the results obtained in Part II were dramatically more positive. Once two prior analogs were given, subjects often derived an approximation to the convergence schema as the incidental product of describing the similarities of the analogs; furthermore, the quality of the induced schema was highly predictive of subsequent transfer performance. In addition, the same verbal statements and diagrams that had failed to influence transfer from a single analog proved highly beneficial when paired with two.

These central results, as well as other more detailed findings (e.g., the extreme separation of noticing from application in the case of a diagrammatic analogy presented alone) support our analyses of analogical 
mapping, schema formation, and semantically driven retrieval. A mapping process cannot operate on only a single prior analog to derive a schema; consequently, the most direct mechanism for schema induction is inapplicable. Two analogs, on the other hand, can be mapped together to derive a more general schema; furthermore, any device that highlights the causally relevant correspondences will facilitate abstraction of a more optimal schema. To the extent that the schema implicit in the prior analogs has been made explicit, analysis of a disparate transfer problem may yield semantic retrieval cues that prompt recall of the prior information. Schema induction will thus increase the probability that an analogy will subsequently be noticed; in addition, a problem schema will simplify the process of mapping the prior information with the new problem in order to generate an analogous solution.

It should be noted that in all of our experiments the critical prior analogs were presented in a context in which their problem-oriented character was incidental. Subjects were never explicitly encouraged to use the stories to learn about a novel kind of problem. In many situations, such as an instructional context, more directive guidance in the application of an analogy is often given. It is quite likely that more intentional learning procedures could improve transfer performance in our paradigm. In particular, explicit guidance might facilitate transfer from a single analog. In the absence of such guidance, failure to derive a general schema from a single instance may only reflect appropriate conservatism; without either further examples or direct instruction, the person may have no principled way to isolate the essential causal aspects of the situation.

Given the difficulty of schema abstraction from a single analog (at least without the guidance of a teacher), one might ask how anyone could spontaneously notice an analogy between one initial analog and a semantically remote transfer problem. After all, we obtained consistent estimates that about $20 \%$ of our subjects solved the radiation problem by using the military story without receiving an explicit suggestion to do so. In part, this minority may simply consist of subjects sensitive to demand characteristics that suggest the two parts of the experiment might be related. However, there are more basic reasons why at least a few people may spontaneously represent the initial story in a way that would enable subsequent transfer. Our subjects may have often brought relevant prior knowledge to the experiment. One subject in an earlier experiment (Gick \& Holyoak, 1980) immediately mapped the story analog with another, previously known convergence problem (in which multiple weak lasers were used to fuse the filament of a lightbulb without breaking the surrounding glass). While few subjects are likely to be reminded of such a complete analogy to the story, many may bring some type of prior knowledge to bear on it. For example, college students are likely to already 
know the abstract concept of "force," and a few may spontaneously encode a military attack as an instance of the application of force. If such subjects later see the radiation problem as one involving a search for a method of applying force, this shared semantic component may trigger recall of the prior story and initiation of a mapping process. More generally, if a current situation can be related to prior knowledge, so that its causally relevant aspects are encoded at an abstract level, then the situation has the potential to be subsequently related to a new analog from a remote domain. Because experts tend to encode problems at a relatively abstract level (Chi, Feltovich, \& Glaser, 1981), it follows that expert knowledge of one domain should tend to enable analogical transfer to another. (See Holyoak, in press, for discussion of the relationship between analogical thinking and problem-solving expertise.)

Given our emphasis on schema induction as a basis for analogical transfer, it is worth considering what, if anything, specific analogs can add to a general schema. It might seem that if a schema could be somehow directly taught, examples would not be of any use at all. However, the present study provides evidence against this hypothesis. The provision of a verbal statement of the convergence principle without any accompanying story (Experiment 2) could be viewed as an attempt to teach the schema directly. The total percentage of subjects producing the target solution in this condition was not exceptionally high (66\%), at least when compared to the levels of transfer obtained in the later experiments with multiple analogs. This might seem to contradict our prediction that reasoning from a schema will be more effective than reasoning from an analog. However, the discrepancy is only apparent. Our prediction refers to the benefit provided by a schema abstracted from specific analogs; there is no reason to assume that the verbal statement used in Experiment 2 conveys the same information as could be induced from the story analogs. Indeed, the verbal principle does not in fact allow as complete a mapping with the radiation problem as do the stories. For example, the principle refers to "accomplishing some purpose," whereas the corresponding core idea in the stories is the more specific relation of "overcoming." In addition, the stories may allow a more detailed mapping that generates information about how the abstract convergence solution could be realized in a medical situation. For instance, the use of multiple hoses in "Red Adair" could suggest the idea of introducing multiple rayemitting machines.

More generally, the more detailed information implicit in the representation of a specific analog may in part account for the intuitive view that examples are often critical for learning; for a teacher to simply "explain the principle" is not enough. To be put to use, a general schema must always be instantiated as a set of specific concepts appropriate to a 
particular domain; an example, even from a different domain, may suggest how the process of instantiation could proceed. Furthermore, in complex domains much of the detailed knowledge shared by experts, particularly procedural knowledge, is likely to be implicit and not easily verbalized. A teacher may therefore have difficulty explicitly teaching such knowledge. However, by presenting the student with selected examples, the knowledge may be conveyed implicitly.

\section{Toward a Computational Model}

A great deal remains to be learned before it will be possible to formulate a computationally adequate model of analogical thinking. Without such a model, it is difficult to know how to optimize people's use of analogies. For example, Polya (1957) suggests searching for similar examples as a problem-solving heuristic; a worthy goal without doubt, but how to find the relevant examples and apply them? Perhaps analogical thinking can be developed by appropriate training; however, this educational goal is handicapped by our lack of knowledge regarding the essential processes underlying the skill. The mapping process presents particularly formidable problems that must be solved in order to construct a process model of analogical thinking, and computational implementations have tended to invoke algorithms that would unduly strain the capacity limits of human working memory. For example, Evan's (1968) program to solve proportional analogies between geometrical figures finds the optimal level of abstraction for representing the figures by computing all possible levels.

A related mapping problem arises from the fact that the number of potential pairings between two sets of concepts quickly becomes very large as the number of concepts in each set grows. Winston's (1980) program to compare story scenarios must either test all possible matches, or else prune away all except those between concepts that share some arbitrary feature (e.g., characters of the same gender). As Winston notes, this algorithm will never discover a male Cinderella. Psychological process models must strive to capture the remarkable flexibility that people exhibit in their use of analogy. The curtains have scarcely parted on this particular window to the mind.

\section{APPENDIX 1 \\ ANALOG TO THE CORD PROBLEM (EXPERIMENT 1)}

\section{The Birthday Party}

It was Jane's sixth birthday and her mother wanted to make it a very special day for her. So she organized a big surprise party and invited the neighborhood children without Jane knowing about it. The plan was that the mother who usually picked up Jane after school would be late in order 
to allow the children time to arrive before Jane. The big day finally arrived. Everything was just about ready, and it was fifteen minutes before the children were supposed to come. Jane's mother was putting the final touches on the decorations for the party room. She was covering the walls and ceiling with balloons and party streamers made of crepe paper and ribbons. Jane's mother was finishing up a decoration pattern. Two final pieces were left that were dangling from the wood paneling above. She had originally planned to knot these two pieces of ribbon together in order to attach balloons to them. However, whenever she grabbed the end of one ribbon, colored blue, she was not able to grasp the other ribbon, colored pink, at the same time. The ribbons simply were not long enough to be knotted together in this way. It seemed that she would have to abandon her final bit of decoration.

Jane's mother was just about to give up when she had an idea. She took the pair of scissors that she had been using to cut the various ribbons and crepe paper, and attached the scissors to the end of the ribbon. Next, she took hold of the scissors, and pointing them in the direction of the pink ribbon, swung them vigorously so that this blue ribbon now swayed alternately between the pink ribbon and a nearby wall. She then ran quickly and took the end of the dangling pink ribbon and walked as close to the swinging blue ribbon as possible without letting go of the pink ribbon. She then waited until the swinging blue ribbon came her way and caught it on the upswing. While still holding the pink ribbon, she then removed the scissors from the other blue ribbon and knotted the two ribbons together. Jane's mother just managed to attach all her balloons on these ribbons, completing her decorations, before the children started pouring in. Soon Jane arrived and was genuinely surprised. The party was a great success, and all the mothers complemented Jane's mother on the decorations.

\section{APPENDIX II \\ FOUR ANALOGS TO THE RADIATION PROBLEM}

\section{The General}

A small country was ruled from a strong fortress by a dictator. The fortress was situated in the middle of the country, surrounded by farms and villages. Many roads led to the fortress through the countryside. A rebel general vowed to capture the fortress. The general knew that an attack by his entire army would capture the fortress. He gathered his army at the head of one of the roads, ready to launch a full-scale direct attack. However, the general then learned that the dictator had planted mines on each of the roads. The mines were set so that small bodies of men could pass over them safely, since the dictator needed to move his troops and workers to and from the fortress. However, any large force 
would detonate the mines. Not only would this blow up the road, but it would also destroy many neighboring villages. It therefore seemed impossible to capture the fortress.

However, the general devised a simple plan. He divided his army into small groups and dispatched each group to the head of a different road. When all was ready he gave the signal and each group marched down a different road. Each group continued down its road to the fortress so that the entire army arrived together at the fortress at the same time. In this way, the general captured the fortress and overthrew the dictator.

\section{The Commander}

A military government was established after the elected government was toppled in a coup. The military imposed martial law and abolished all civil liberties. A tank corp commander and his forces remained loyal to the overthrown civilian government. They hid in a forest waiting for a chance to launch a counterattack. The commander felt he could succeed if only the military headquarters could be captured. The headquarters was located on a heavily guarded island situated in the center of a lake. The only way to reach the island was by way of several pontoon bridges that connected it to the surrounding area. However, each bridge was so narrow and unstable that only a few tanks could cross at once. Such a small force would easily be repulsed by the defending troops. The headquarters therefore appeared invincible.

However, the tank commander tried an unexpected tactic. He secretly sent a number of tanks to locations near each bridge leading to the island. Then under cover of darkness the attack was launched simultaneously across each bridge. All of the groups of tanks arrived on the island together and immediately converged on the military headquarters. They managed to capture the headquarters and eventually restore the civilian government.

\section{Red Adair}

An oil well in Saudi Arabia exploded and caught fire. The result was a blazing inferno that consumed an enormous quantity of oil each day. After initial efforts to extinguish it failed, famed firefighter Red Adair was called in. Red knew that the fire could be put out if a huge amount of fire retardant foam could be dumped on the base of the well. There was enough foam available at the site to do the job. However, there was no hose large enough to put all the foam on the fire fast enough. The small hoses that were available could not shoot the foam quickly enough to do any good. It looked like there would have to be a costly delay before a serious attempt could be made.

However, Red Adair knew just what to do. He stationed men in a circle 
all around the fire, with all of the available small hoses. When everyone was ready all of the hoses were opened up and foam was directed at the fire from all directions. In this way a huge amount of foam quickly struck the source of the fire. The blaze was extinguished, and the Saudis were satisfied that Red had earned his three million dollar fee.

\section{The Fire Chief}

One night a fire broke out in a wood shed full of timber on $\mathrm{Mr}$. Johnson's place. As soon as he saw flames he sounded the alarm, and within minutes dozens of neighbors were on the scene armed with buckets. The shed was already burning fiercely, and everyone was afraid that if it wasn't controlled quickly the house would go up next. Fortunately, the shed was right beside a lake, so there was plenty of water available. If a large volume of water could hit the fire at the same time, it would be extinguished. But with only small buckets to work with, it was hard to make any headway. The fire seemed to evaporate each bucket of water before it hit the wood. It looked like the house was doomed.

Just then the fire chief arrived. He immediately took charge and organized everyone. He had everyone fill their bucket and then wait in a circle surrounding the burning shed. As soon as the last man was prepared, the chief gave a shout and everyone threw their bucket of water at the fire. The force of all the water together dampened the fire right down, and it was quickly brought under control. Mr. Johnson was relieved that his house was saved, and the village council voted the fire chief a raise in pay.

\section{REFERENCES}

Bishop, Y. M. M., Fienberg, S. E., \& Holland, P. W. Discrete multivariate analysis: Theory and practice. Cambridge, MA: MIT Press, 1975.

Black, M. Metaphor. In M. Black, Models and metaphors. Ithaca, NY: Cornell Univ. Press, 1962.

Chi, M. T. H., Feltovich, P. I., \& Glaser, R. Categorization and representation of physics problems by experts and novices. Cognitive Science, 1981, 5, 121-152.

Duncker, K. A qualitative (experimental and theoretical) study of productive thinking (solving of comprehensible problems). Journal of Genetic Psychology, 33, 1926, $642-708$.

Duncker, K. On problem solving. Psychological Monographs, 1945, 58 (Whole No. 270).

Evans, T. G. A. A program for the solution of geometric-analogy intelligence test questions. In M. Minsky (Ed.), Semantic information processing. Cambridge, MA: MIT Press, 1968.

Gentner, D., \& Gentner, D. R. Flowing waters or teeming crowds: Mental models of electricity. In D. Gentner \& A. Stevens (Eds.), Mental models. Hillsdale, NJ: Erlbaum, in press.

Gick, M. L., \& Holyoak, K. J. Analogical problem solving. Cognitive Psychology, 1980, 12, 306-355.

Hays, W. L. Statistics for the social sciences New York: Holt, Rinehart \& Winston, 1973. 2nd ed. 
Hesse, M. B. Models and analogies in science. Notre Dame, IN: Univ. of Notre Dame Press, 1966.

Holyoak, K. J. Analogical thinking and human intelligence. In R. J. Sternberg (Ed.), Advances in the psychology of human intelligence. Hillsdale, NJ: Erlbaum, in press. Vol. 2.

Homa, D., \& Vosburgh, R. Category breadth and the abstraction of prototypical information. Journal of Experimental Psychology: Human Learning and Memory, 1976, 2, $322-330$.

Jaynes, J. The origin of consciousness in the breakdown of the bicameral mind. Boston: Houghton Mifflin, 1976.

Kintsch, W., \& van Dijk, T. A. Toward a model of text comprehension and production. Psychological Review, 1978, 85, 363-394.

Kohler, W. Dynamics in psychology. New York: Liveright, 1940.

Mackie, J. L. The cement of the universe. London/New York: Oxford Univ. Press, 1974.

Maier, N. Reasoning in humans. I. On direction. Journal of Comparative Psychology, 1930, $10,15-43$.

Maier, N. Reasoning in humans. II. The solution of a problem and its appearance in consciousness. Journal of Comparative Psychology, 1931, 12, 181-194.

Miller, G. A. Images and models, similes and metaphors. In A. Ortony (Ed.), Metaphor and thought. London/New York: Cambridge Univ. Press, 1979.

Moore, J., \& Newell, A. How can MERLIN understand? In L. W. Gregg (Ed.), Knowledge and cognition. Potomac, MD: Erlbaum, 1973.

Peterson, M. J., Meagher, R. B., Chait, H., \& Gillie, S. The abstraction and generalization of dot patterns. Cognitive Psychology, 1973, 4, 378-398.

Polya, G. How to solve it. Princeton, NJ: Princeton Univ. Press, 1957.

Posner, M. I., \& Keele, S. W. On the genesis of abstract ideas. Journal of Experimental Psychology, 1968, 77, 353-363.

Reed, S. K., Ernst, G. W., \& Banerji, R. The role of analogy in transfer between similar problem states. Cognitive Psychology, 1974, 6, 436-450.

Schon, D. A. Generative metaphor: A perspective on problem-setting in social policy. In A. Ortony (Ed.), Metaphor and thought. London/New York: Cambridge Univ. Press, 1979.

Shustack, M., \& Anderson, J. R. Effects of analogy to prior knowledge on memory for new information. Journal of Verbal Learning and Verbal Behavior, 1979, 18, 565-583.

Sternberg, R. J. Intelligence, information processing, and analogical reasoning: The componential analysis of human abilities. Hillsdale, NJ: Erlbaum, 1977.

Thorndyke, P. W. Cognitive structures in comprehension and memory of narrative discourse. Cognitive Psychology, 1977, 9, 77-110.

Tversky, A. Features of similarity. Psychological Review, 1977, 84, 327-352.

van Dijk, T. A. Macrostructures. Hillsdale, NJ: Erlbaum, 1980.

Winston, P. H. Learning and reasoning by analogy. Communications of the ACM, 1980, 23, 689-703.

\section{REFERENCE NOTES}

1. Gick, M. L. Analogical reasoning and schema formation. PhD dissertation, University of Michigan, 1981.

2. Weitzenfeld, J., \& Klein, G. A. Analogical reasoning as a discovery logic. Technical Report TR-SCR-79-5, Klein Associates, Yellow Springs, OH, 1979.

3. Fried, L. S., \& Holyoak, K. J. Induction of category distributions: A framework for classification learning. Michigan/Chicago Cognitive Science Technical Report 38, 1982.

4. Nitsch, K. E. Structuring decontextualized forms of knowledge. PhD dissertation, Vanderbilt University, 1977.

Accepted March 23, 1982. 